\title{
Empatía y Estrategías de Afrontamiento como Predictores del Bienestar en Estudiantes Universitarios Españoles
}

\section{Josep Gustems Carnicer ${ }^{1}$ y Caterina Calderón ${ }^{2}$,}

${ }^{1}$ Departamento de Didáctica Musical y Corporal, Universidad de Barcelona

${ }^{2}$ Departamento de Personalidad, Evaluación y Tratamiento Psicológico, Universidad de Barcelona

\section{España}

Correspondencia: Josep Gustems Carnicer. Pg. Vall d'Hebron 171, edificio llevant $3^{\text {a }}$ planta. (08035) Barcelona. Spain. E-mail: jgustems@ub.edu

(C) Education \& Psychology I+D+i and Ilustre Colegio Oficial de Psicología de Andalucía Oriental (Spain) 


\section{Resumen}

Introducción. La empatía y las estrategias de afrontamiento han sido asociadas firmemente con el bienestar psicológico percibido por los estudiantes. El objetivo del presente estudio fue analizar la relación entre la empatía, las estrategias de afrontamiento y el malestar psicológico entre estudiantes universitarios.

Método. Se realizó un estudio descriptivo, transversal. Noventa estudiantes de educación contestaron algunos cuestionarios (Brief Symptom Inventory, Coping Responses Inventory-Adult y Empathy Affective and Cognitive Test) como parte de un cribado general de salud.

Resultados. Los estudiantes con alto riesgo de presentar malestar psicológico obtuvieron puntuaciones significativamente más altas en estrés empático y estrategias de afrontamiento evitativas. El malestar psicológico mostró una correlación positiva y significativa con la descarga emocional, la evitación cognitiva, la búsqueda de recompensas alternativas y la aceptación/resignación, y una correlación negativa con la resolución de problemas. La descarga emocional y la resolución de problemas explicaron el $29.4 \%$ de la varianza del distrés psicológico en estudiantes de educación.

Conclusión. Reconocer las emociones de uno mismo y comprender los estados emocionales y las intenciones de los demás es beneficioso para el estudiante. El desarrollo de los propios recursos y estrategias de afrontamiento permitirá a los estudiantes, futuros maestros adaptarse a situaciones estresantes que puede encontrarse en el contexto educativo.

Palabras clave: Empatía, estrategias de afrontamiento, malestar psicológico, estudiantes de educación. 


\title{
Empathy and Coping Strategies as Predictors of Well-being in Spanish University Students
}

\begin{abstract}
Introduction. Empathy and coping strategies have been shown to be closely associated with the perceived psychological well-being of students. The aim of the present study was to analyze the relationship between empathy and coping strategies and psychological distress among students in teacher education.
\end{abstract}

Method. A cross-sectional study. Ninety teacher education students completed questionnaires (Brief Symptom Inventory, Coping Responses Inventory-Adult and Empathy Affective and Cognitive Test) as part of general health screening.

Results. Students with high risk of exhibiting psychological distress obtained significantly higher scores in empathy stress and avoidance coping strategies. Psychological distress showed a significant positive correlation with emotional discharge, cognitive avoidance, seeking alternative rewards and acceptance/resignation, and negative correlation with problem solving. Emotional discharge and problem solving explain $29.4 \%$ of the variance of psychological distress in teacher education students.

Conclusion. Recognizing one's emotions and understand the emotional states and intentions of others is beneficial to the student. The development of own resources and coping strategies will enable students adapt to stressful situations that can be found educational context.

Keywords: Empathy, coping strategies, psychological distress, teacher education students. 


\section{Introducción}

La empatía y las estrategias de afrontamiento se han relacionado estrechamente con la percepción de bienestar psicológico entre los estudiantes (Dyson \& Renk, 2006; Shanafelt, West, Zhao, Novotny, Kolars, Habermann \& Sloan, 2005). Ambas son habilidades importantes que facilitan que el estudiante finalice con éxito sus estudios (Oceja, López-Pérez, Ambrona \& Férnandez-Pinto, 2009).

Eisenberg, Fabes, Murphy, Karbon, Maszk, Smith, O’Boyle y Suh (1994) definen la empatía como una respuesta afectiva que permite la comprensión del estado o condición emocional del otro, es un concepto multidimensional que incluye aspectos cognitivos y afectivos (Hoffman, 2000). En la dimensión cognitiva de la empatía tendríamos la capacidad intelectual o imaginativa de ponerse en el lugar de la otra persona (adopción de perspectivas), y de reconocer y comprender los estados emocionales e intenciones de los demás (comprensión empática). La dimensión afectiva incluye la capacidad de compartir, tanto las emociones positivas (alegría empática) como las emociones negativas de otra persona (estrés empático).

Uno de los factores más importantes para asegurar el bienestar psicológico es el establecimiento de buenas relaciones con los demás (Acun-Kapikiran, 2011; Shanafelt \& West, 2007). En este sentido, la empatía facilita el contacto interpersonal satisfactorio (Granello, 1999), el incremento de la popularidad social (Vitaglione \& Barnett, 2003) y la conducta prosocial (Carlo, Allen \& Buhman, 1999), y funciona como un amortiguador en la disminución de la agresión (Björkqvist, Österman \& Kaukiainen, 2000).

La empatía es importante para los futuros maestros, pues permite comprender las necesidades del alumno y ofrecer una atención adecuada y de calidad. Sin embargo, puntuaciones muy elevadas en estrés empático dificultarían la objetividad profesional e interferirían negativamente en la salud psicológica de los educadores (Batson, Batson, Brummett, Shaw \& Aldeguer, 1995), llegando incluso a tomar decisiones erróneas o injustas (Oceja, 2008).

La empatía facilita la autorregulación emocional, así los estudiantes capaces de regular sus emociones y comportamiento emocional experimentarán probablemente más empatía que distrés personal, y serán más capaces de desarrollar estrategias de afrontamiento más adaptadas al contexto educativo. 
Las estrategias de afrontamiento son un conjunto de acciones y cogniciones que capacitan al estudiante a tolerar, evitar o minimizar los efectos producidos por una situación estresante (Parker \& Endler, 1992). Folkman y Lazarus (1986) propusieron dos estilos de afrontamiento: el afrontamiento activo focalizado en el problema con el objetivo de modificar la situación problemática para hacerla menos estresante, y el afrontamiento evitativo focalizado en la emoción, encaminado a reducir la tensión, la activación fisiológica y la reacción emocional (Frydenberg, Lewis, Kennedy, Ardila, Frindte \& Hannoun, 2003; Plancherel, Bolognini \& Halfon, 1998). El afrontamiento aproximativo está asociado con índices positivos de bienestar psicológico (Parson, Frydenberg \& Poole, 1996), mientras que el estilo de afrontamiento evitativo focalizado en la emoción está asociado con la percepción de mayor malestar psicológico (Kirchner, Forns, Amador \& Muñoz, 2010). Un uso adecuado de estas estrategias de afrontamiento permitirá al alumno adaptarse mejor a las situaciones de estrés académico y laboral.

En este sentido, el ingreso a la universidad se ha considerado tradicionalmente una etapa caracterizada por muchos cambios que pueden ocasionar estrés en el estudiante (Arnett, 2000). Esto se debe a que en este periodo coinciden diversos factores: el proceso de separación de la familia, la incorporación al mercado laboral, la adaptación a un medio poco habitual y las altas cargas de trabajo (Beck, Taylor \& Robbins, 2003). Las exigencias psicosociales derivadas de este proceso pueden repercutir en el bienestar psicológico del estudiante, mostrando una peor regulación emocional o empatía (Carr, Colthurst, Coyl, \& Elliott, 2012), un uso excesivo de estrategias de afrontamiento evitativas (Wong, Bheung, Chan, Ma \& Tang, 2006), incremento de la ansiedad y la depresión (Dahlin, Joneborg, Wong, Bheung, Chan, Ma \& Tang, 2006; Runeson, 2005), y mostrar menor actividad y una productividad más baja (Chandavarkar, Azzam \& Mathews, 2007).

\section{Objetivo del presente estudio}

Teniendo en cuenta que existen numerosos estudios que señalan altos niveles de estrés entre los estudiantes (Leung, Mak, Chui, Chiang \& Lee, 2009; Travers \& Cooper, 1996) y los educadores (Chan, Lai, Ko \& Boey, 2000; Leung, Siu \& Spenctor, 2000) es importante conocer en qué medida la capacidad de autorregulación y la estrategias de afrontamiento influirán en la percepción de malestar psicológico en los estudiantes. Por lo tanto, el objetivo de esta investigación es examinar la relación existente entre la empatía, las estrategias de afrontamiento y el malestar psicológico en estudiantes de maestro españoles. 


\section{Método}

\section{Participantes}

Una muestra de 90 estudiantes de la Universidad de Barcelona participaron en este estudio. Todos los participantes completaron los cuestionarios durante el horario de clases como parte de una revisión general de salud administrada en los grupos de segundo curso, todos ellos de ciencias sociales. Los estudiantes tenían edades entre los 19 y los 42 años $(M=23.2$; $S D=4.82)$, un $98 \%$ fueron mujeres.

\section{Instrumentos}

Brief Symptom Inventory (BSI) (Derogatis \& Spencer, 1982). Se aplicó la adaptación española (Ruipérez, Ibáñez, Lorente, Moro \& Ortet, 2001) del Brief Symptom Inventory (BSI). El BSI es un cuestionario auto-informe de 53 items diseñado para evaluar una amplia gama de síntomas psicológicos en adultos mediante una escala Likert de cinco puntos que va de uno (total ausencia de síntoma) a cinco (total presencia del síntoma). La escala proporciona nueve grupos de síntomas (somatización, obsesividad-compulsividad, sentitividad interpersonal, depresión, ansiedad, hostilidad, ansiedad fóbica, ideación paranoide y psicoticismo). El análisis se basó en el Índice de Severidad Global, que proporciona una medida segura y válida de malestar psicológico combinando información del número de síntomas que la persona presenta y la intensidad percibida del malestar psicológico. La fiabilidad test-retest va desde 0.78 a 0.90. El alfa de Cronbach puntuó de 0.81 a 0.90.

Empathy affective and cognitive Test (TECA) (López-Perez, Férnandez-Pinto \& AbadGarcía, 2008). El TECA es una escala de autoinforme con 33 items diseñada para evaluar los aspectos cognitivos y afectivos de la empatía en adultos mediante una escala Likert de cinco puntos que van de uno (total desacuerdo) a cinco (muy de acuerdo). La escala proporcina cinco indices: adopción de perspectivas, comprensión empática, estrés empático, alegría empática y un índice global de empatía. La fiabilidad test-retest fue de 0.86. El alfa de Cronbach puntuó de 0.70 a 0.86 .

Coping Responses Inventory-Adult form (CRI-A) (Moos, 1993). Para evaluar las estrategias de afrontamiento, se usó la adaptación española del Coping Responses Inventory-Adult Form (CRI-A). El CRI-Adulto es una escala auto-informada de 48 ítems que evalua las res- 
puestas de afrontamiento a las experiencias vitales estresantes en adultos a partir de 18 años de edad. Estas respuestas son medidas por escalas con 6 ítems que utilizan una escala Likert de cuatro puntos desde 0 ("nada en absoluto") a 3 ("con bastante frecuencia"). Cuatro escalas miden e afrontamiento aproximativo: análisis lógico, reevaluación positiva, búsqueda de guía y soporte, y resolución de problemas. Análisis Lógico: una estrategia cognitiva diseñada para compreder y prepararse uno mismo mentalmente para afrontar estresores y sus consecuencias. Reevaluación Positiva: trata de reconstruir el problema en un modo positivo mientras se acepta la realidad de la situación. Búsqueda de guía y soporte: supone intentos conductuales para busca información, guía o soporte para hacer frente al estresor. Resolución de problemas: denota intentos conductuales para afrontar directamente el problema y solucionarlo. Las cuatro escalas restantes evaluan afrontamiento evitativo: evitación cognitiva, aceptación/resignación, búsqueda de recompensas alternativas, y descarga emocional. Evitación Cognitiva: se refiere a intentos cognitivos de evitar pensar en el problema o en el estresor. Aceptación o Resignación: se refiere a intentos cognitivos para responder a un problema aceptándolo y resignándose a él, porque nada puede hacerse. Búsqueda de Recompensas Alternativas: denota tentativas conductuales para mitigar el efecto del estrés causado por un problema buscando nuevas formas de satisfacción. Descarga Emocional: se refiere a intentos conductuales para reducer la tension expresando sentimientos negativos. La adaptación española del CRI-A presenta Buena fiabilidad, con una alfa de Cronbach entre 0.81 y 0.90. El inventario es ampliamente usado en contextos educativos y sociales (Kirchner, Forns, Muñoz \& Pereda, 2008).

\section{Procedimiento}

Los estudiantes completaron los cuestionarios durante el periodo regular de clases con el permiso de sus profesores. Los estudiantes también fueron informados sobre el estudio y los procedimentos de evaluación. A todos se les informó de que su participación era complemente voluntaria y que podían escoger no participar o no responder algunas preguntas específicas que les incomodasen y todos ellos firmaron el consentimiento informado. Se garantizó el anonimato y la confidencialidad mediante el uso de códigos para los datos obtenidos. El estudio se enmarca dentro de las directrices del Informe Belmont (1978) y del Código de Buenas Prácticas en Investigación de la Universidad de Barcelona (2010).

Los estudiantes fueron clasificados según el alto o bajo riesgo de presentar malestar psicológico mediante el criterio propuesto por Derogatis (1993). Los estudiantes cuyas pun- 
tuaciones corresponden al percentil 90 o superior en el índice de severidad global GSI fueron considerados que tenían alto riesgo de presentar malestar psicológico; los participantes que puntuaron en el rango normal fueron considerados asintomáticos o con bajo riesgo de presenter malestar psicológico. Globalmente, los resultados fueron estratificados según edad y sexo.

\section{Análisis Estadísticos}

Se describieron las características de los estudiantes con alto o bajo riesgo de presentar malestar psicológico, en el caso de variables cuantitativas, con porcentajes y desviaciones estándar; se usaron frecuencias y porcentajes para las variables cualitativas. Para analizar las diferencias entre ambos grupos, se aplicó la prueba Mann-Whitney U y la corrección Monte Carlo a las siguientes variables: escalas de estrategias de afrontamiento (análisis lógico, reevaluación positiva, búsqueda de guía y soporte, resolución de problemas, evitación cognitiva, aceptación/resignación, búsqueda de recompensas alternativas, y descarga emocional) y las escalas de empatía (adopción de perspectivas, comprensión empática, estrés emocional, alegría empática y el índice global de empatía). Se usó una regresión lineal para predecir la relación entre la presencia de malestar psicológico y estrategias de afrontamiento y empatía en estudiantes. Se utilizó el Statistical Package for Social Sciences (SPSS), versión 16.0, para el procesamiento de los datos. En todos los casos, la significación estadística se fijó en $p<.05$.

\section{Resultados}

\section{Estrategias de afrontamiento y escalas de empatía para estudiantes}

Los estudiantes con un alto riesgo de presentar malestar psicológico puntuaron significativamente alto en cuatro subescalas del CRI-A (Tabla 1): evitación cognitiva $(U=552.5$, $p=.015)$, aceptación/resignación $(U=511.5, p=.005)$, búsqueda de recompensas alternativas $(U=544.5, p=.012)$, y descarga emocional $(U=434.5, p=.0001)$. En las medidas de empatía (TECA), los estudiantes con alto riesgo de presentar síntomas de malestar psicológico puntuaron más alto en estrés empático $(U=581.0, p=.031)$ que aquellos que no mostraron tales síntomas.

Tabla 1. Comparación entre estudiantes con bajo o alto riesgo de presentar malestar psicológico con respecto a la empatía (TECA) y las estrategias de afrontamiento (CRI-A) 


\begin{tabular}{|c|c|c|c|c|c|c|c|c|}
\hline & \multicolumn{2}{|c|}{$\begin{array}{l}\text { Estudiantes con } \\
\text { bajo riesgo de } \\
\text { malestar psicológi- } \\
\text { co }(\mathrm{n}=45)\end{array}$} & \multicolumn{2}{|c|}{$\begin{array}{l}\text { Estudiantes con alto } \\
\text { riesgo de malestar } \\
\text { psicológico }(n=45)\end{array}$} & \multicolumn{2}{|c|}{$\begin{array}{l}\text { Prueba de Mann- } \\
\text { Whithney }\end{array}$} & \multicolumn{2}{|c|}{$\begin{array}{l}95 \% \text { Intervalo de } \\
\text { Confianza }^{\text {a }}\end{array}$} \\
\hline & Media & $\mathrm{DE}$ & Media & $\mathrm{DE}$ & $\mathrm{U}$ & Sig (bil.) & Inferior & Superior \\
\hline \multicolumn{9}{|l|}{ TECA } \\
\hline Adopción de Perspectivas & 51.15 & $(8.37)$ & 50.20 & $(8.14)$ & 753.0 & .653 & .641 & .337 \\
\hline Comprensión Empática & 58.00 & $(10.95)$ & 57.97 & $(8.73)$ & 782.0 & .866 & .857 & .447 \\
\hline Estrés Empático & 51.67 & $(9.35)$ & 55.50 & $(8.82)$ & 581.0 & .031 & .027 & .019 \\
\hline Alegría Empática & 57.97 & $(10.31)$ & 60.62 & $(9.46)$ & 736.5 & .542 & .529 & .279 \\
\hline Índice Global de Empatía & 59.02 & $(10.66)$ & 58.22 & $(10.37)$ & 670.5 & .218 & .207 & .118 \\
\hline \multicolumn{9}{|l|}{ CRI-A } \\
\hline Análisis Lógico & 49.37 & $(8.73)$ & 51.50 & $(6.35)$ & 669.5 & .200 & .189 & .210 \\
\hline Reevaluación Positiva & 51.90 & $(9.48)$ & 48.55 & $(10.54)$ & 673.0 & .217 & .206 & .227 \\
\hline $\begin{array}{l}\text { Búsqueda de Guía y So- } \\
\text { porte }\end{array}$ & 46.45 & $(7.72)$ & 46.92 & $(8.11)$ & 753.5 & .656 & .643 & .668 \\
\hline Resolución de Problemas & 50.15 & $(8.42)$ & $47, .55$ & (7.58) & 652.5 & .151 & .142 & .160 \\
\hline Evitación Cognitiva & 48.72 & $(9.60)$ & 54.57 & $(8.20)$ & 552.5 & .015 & .012 & .018 \\
\hline Aceptación/Resignación & 42.95 & $(8.28)$ & 48.32 & $(9.53)$ & 511.5 & .005 & .004 & .007 \\
\hline $\begin{array}{l}\text { Búsqueda de Recompensas } \\
\text { Alternativas }\end{array}$ & 49.70 & $(7.97)$ & 53.92 & $(7.03)$ & 544.5 & .012 & .009 & .014 \\
\hline Descarga Emocional & 45.67 & $(4.92)$ & 50.65 & $(6.64)$ & 434.5 & .001 & .000 & .001 \\
\hline
\end{tabular}

\footnotetext{
${ }^{\text {a }}$ Monte Carlo Sig. (2-colas).
}

\section{Correlaciones entre malestar psicológico, estrategias de afrontamiento y escalas de empatía}

Los resultados en la Figura 1 revelan que el afrontamiento evitativo mostró una correlación positiva significativa con el malestar psicologico. En concreto, este fue el caso de la evitación cognitiva ( $r=.305, p=.006)$, aceptación/resignación $(r=.262, p=.019)$, búsqueda de recompensas alternativas $(r=.274, p=.014)$, y descarga emocional $(r=.489, p=.001)$. Hubo también una correlación negativa significativa entre el malestar psicológico y la resolución de problemas $(r=-.255, p=.022)$. Sin embargo, no se encontraron diferencias estadísticas significativas en relación al análisis lógico $(r=-.047, p=.676)$, reevaluación positiva $(r=$ -.207, $p=.066)$, o búsqueda de guía y soporte $(r=-.059, p=.605)$. No hubo correlaciones significativas entre las puntuaciones de las escalas de empatía y la presencia de malestar psicológico en los estudiantes (adopción de perspectivas: $r=-.112, p=.325$; comprensión empá- 
tica: $r=-.081, p=.476$; estrés empático: $r=.213, p=.057$; alegría empática: $r=-1.68$, $p=.136 ; \mathrm{y}$ el índice global de empatía: $r=.072, p=.528)$.

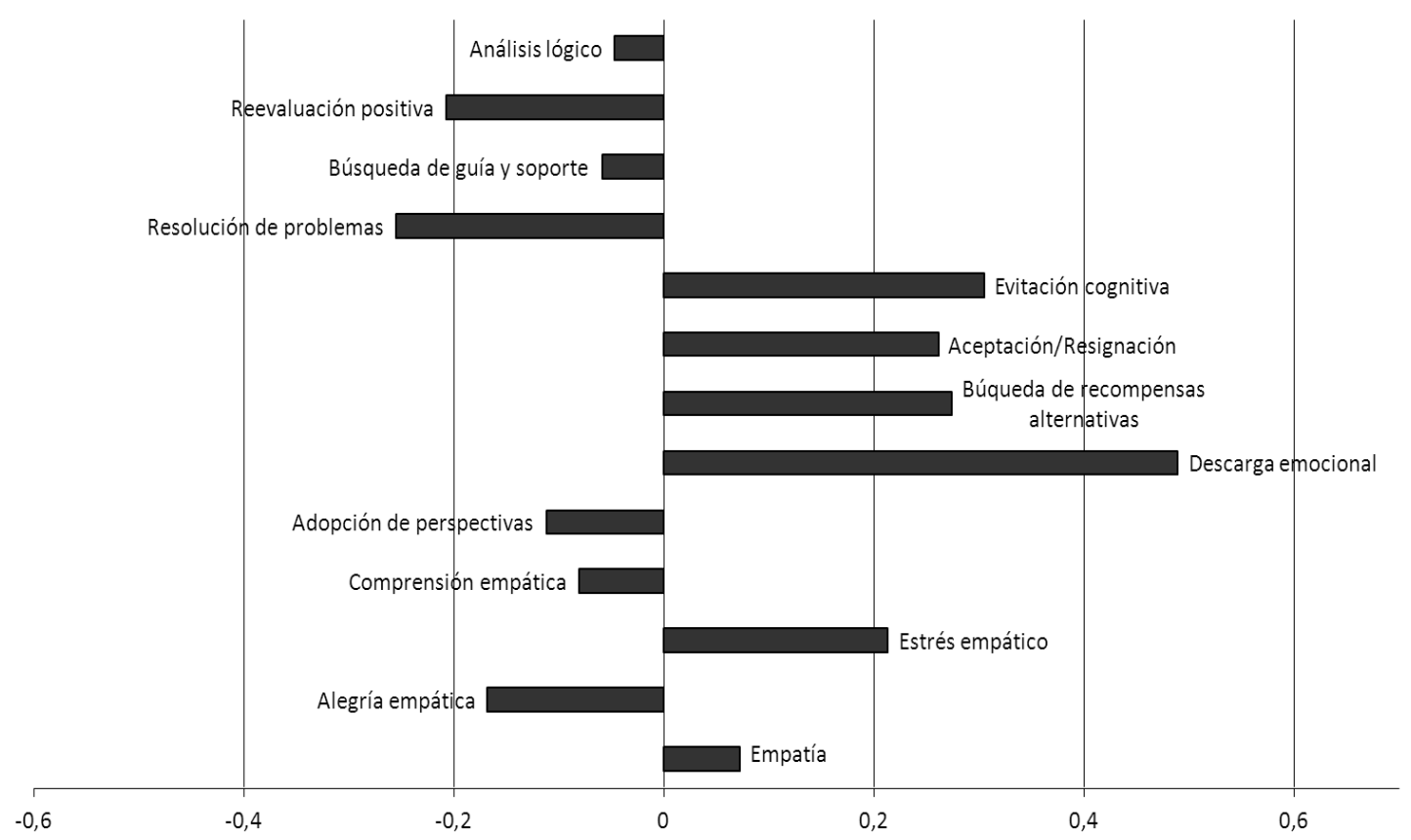

Figura 1. Correlaciones entre estrategias de afrontamiento, empatía y presencia de malestar psicológico en estudiantes

\section{Variables que explican el malestar psicológico en los estudiantes}

Los resultados de la regresión lineal indicaron que la descarga emocional explicó el $22.9 \%$ de la varianza en el malestar psicológico $(F=24.530, p=.0001 ; 95 \%$ Intervalo de Confianza $=.073-2.330)$, mientras que la descarga emocional junto con la resolución de problemas explicó el 29.4\% de la varianza $(F=17.462, p=.0001$; 95\% Intervalo de Confian$\mathrm{za}=1.008-2.318$ para la descarga emocional y el 95\% Intervalo de Confianza $=-.467--$ .079 para la resolución de problemas), ver Tabla 2.

La siguiente tabla muestra un análisis de regresión lineal para determinar la relación entre el malestar psicológico en estudiantes y las variables independientes estudiadas. 
Tabla 2. Análisis de regresión lineal; $\mathbf{R}$ corregida $\left(\mathbf{R}^{2}\right)$, coeficiente no-estandarizado $(\mathbf{B})$, coeficiente beta estandarizado ( $\beta)$, error estándar; análisis de varianza y nivel de significación.

\begin{tabular}{lccccccc} 
Variables Independientes & Variable Dependiente & $\mathrm{R}^{2}$ & $\mathrm{~B}$ & $\mathrm{SE} B$ & $\beta$ & $\mathrm{F}$ & $\mathrm{p}$ \\
\hline $\begin{array}{l}\text { Modelo 1 } \\
\text { Descarga Emocional }\end{array}$ & Malestar Psicológico & .229 & .712 & .144 & .489 & 24.530 & .0001 \\
\hline $\begin{array}{l}\text { Modelo 2 } \\
\text { Descarga Emocional }\end{array}$ & Malestar Psicológico & .294 & .724 & .138 & .497 & 17.462 & .0001 \\
$\quad \begin{array}{l}\text { Resolución de proble- } \\
\text { mas }\end{array}$ & & -.308 & .108 & -.270 & \\
\hline
\end{tabular}

Variable Dependiente: Malestar Psicológico

Modelo 1. Predictores: (Constante), descarga emocional

Modelo 2. Predictores: (Constante), descarga emocional y resolución de problemas

\section{Discusión}

Los análisis realizados muestran que la empatía y las estrategias de afrontamiento están relacionadas con el bienestar psicológico entre los estudiantes, en la misma línea que otras investigaciones realizadas sobre el tema (Thomas, Dyrbye, Huntington, Lawson, Novotny, Sloan \& Shanafelt, 2007). Nuestros resultados indican que los estudiantes con mayor tendencia a sobreimplicarse en los problemas de los demás (estrés empático) presentan más malestar psicológico. A priori podemos pensar que la empatía es un valor positivo y que los educadores deberían presentar altas puntuaciones en empatía para el desempeño de su profesión, pero tal y como sugieren Eisenberg, Fabes, Guthrie, y Reiser (2000) una excesiva implicación en el afecto negativo (estrés empático) comprometería la objetividad profesional, provocaría aversión, excedería emocionalmente a la persona e interferiría negativamente en su bienestar personal (Johanloo \& Chaedi, 2009). Autores como Miner (2007) ha señalado que la presencia de puntuaciones elevadas en estrés empático indicaría mayor propensión a padecer problemas relacionados con la ansiedad y por tanto facilitarían la aparición del burnout.

Nuestros resultados también revelan una relación entre el malestar psicológico y los estilos de afrontamiento evitativos, tanto conductuales (uso de alcohol u otras drogas evaluado mediante la escala de búsqueda de recompensas alternativas o la descarga emocional para reducir o tolerar mejor el estrés) como cognitivos (evitar pensar sobre el problema o el estresor, o respondiendo a un problema aceptándolo o resignándose al mismo). La relación entre el uso de estrategias de afrontamiento evitativas y el malestar psicológico coincidiría con otros 
autores, que indican una correlación significativa entre el estrés y el afrontamiento evitativo (Dwyer \& Cummings, 2001; Watson \& Sinha, 2008). En un estudio sobre estudiantes universitarios Werch, Bian, Moore, Ames, DiClemente y Weiler (2007) encontraron que el uso de estrategias evitativas estaba asociado tanto con el consumo de alcohol como con el malestar psicológico. Nuestros resultados muestran también que un menor uso de estrategias aproximativas (por ejemplo, tartar de afrontar directamente un problema y resolverlo) está relacionado con un mayor malestar psicológico entre los estudiantes. En esta línea, Mikulic y Crespi (2008) señalan que las estrategias de afrontamiento activas o aproximativas se relacionan con una mejor salud, mientras que las estrategias evitativas lo están con el desarrollo de distintas enfermedades. Observamos, sin embargo que Holahan, Moos, Holahan, Brennan, y Schutte (2005) demostraron que las respuestas de afrontamiento varían dependiendo de la severidad del estresor. A mayor cantidad de sucesos de la vida negativos y estresores crónicos, menor uso de respuestas de aproximación al problema y mayor uso de repuestas de evitación (Mikulic \& Crespi 2008).

Asi mismo, los resultados mostraron que las dos respuestas de afrontamiento que mejor predicen el malestar psicológico en estudiantes fueron el uso excesivo de estrategias evitativas de tipo conductual, tales como la descarga emocional (es decir, intentos de reducir la tensión mediante la expresión de sentimientos negativos) y un uso insuficiente de estrategias activas de aproximación, tales como la resolución de problemas (tentativas conductuales de afrontar directamente un problema y resolverlo). En la línea señalada por otros autores, el estilo de afrontamiento improductivo se relaciona con un bajo bienestar, mientras que un estilo de afrontamiento dirigido a la resolución del problema se relaciona con altos niveles de bienestar (Cross \& Hong, 2012; Fickova \& Korcova, 2000). Estos resultados ponen de manifiesto que, en la muestra de estudiantes universitarios analizada, las estrategias de afrontamiento (como un uso excesivo de descarga emocional y un uso insuficiente de estrategias para intentar solucionar el problema) influirían más que la empatía, en el malestar psicológico percibido por los mismos.

En conclusión, los estudiantes universitarios pueden presentar altos niveles de estrés que disminuyan su rendimiento (Morrison \& O'Connor, 2005) e incrementen la presencia de ansiedad y depresión (Garlow, Rosenberg, Moore, Hass, Koestner, Hendin \& Nemeroff, 2008). Reconocer las propias emociones y comprender los estados emocionales e intenciones de los demás es beneficioso para reducir o tolerar el estrés en el estudiante. El desarrollo de 
recursos propios como las estrategias de afrontamiento también permitirá a los futuros estudiantes de maestro adaptarse a las situaciones de estrés que pueden encontrarse en el contexto laboral educativo, organizar y planificar mejor su material docente, aplicar sus competencias en disciplina y control de clase, y adaptarse mejor a las transiciones y cambios (Jaunitz, Spokane, Lissitz \& Stein, 1986). En concreto, estrategias dirigidas a la organización el tiempo, reevaluación positiva, actividad física, humor, y la relajación (Gardner, 2010) pueden ayudar también a que los futuros maestros se sientan mejor.

Futuras investigaciones deberían estudiar la relación entre la cantidad y el tipo de estresores con las respuestas de afrontamiento en los estudiantes universitarios. También se podría analizar la relación entre el estrés empático, la ansiedad y el burnout en maestros.

Aunque los resultados de este estudio sean relevantes para nuestro campo de conocimiento, existen algunas limitaciones que deben ser consideradas al plantear las conclusiones. Como es notorio, casi todos los participantes fueron mujeres y la muestra estaba formada por estudiantes de una única facultad, por lo que quizás los resultados no son generalizables a otros colectivos. En segundo lugar, los participantes son jóvenes estudiantes de maestro, y los resultados podrían no ser representativos de otros grupos. Finalmente, la naturaleza transversal de los datos no nos permite determinar la causalidad de la relación entre la empatía, las estrategias de afrontamiento y el malestar psicológico.

\section{Referencias}

Acun-Kapikiran, N. (2011). Focus on Positive and Negative Information as the Mediator of the Relationship between Empathy Tendency Guilty and Psychological Well-Being in University Student. Educational Sciences: Theory and Practice, 11(3), 1141-1147.

Arnett, J.J. (2000). Emerging adulthood: A theory of development from the late teen through the twenties. American Psychologist, 55, 469-480. doi: 10.1037/0003-066X.55.5.469

Batson, C.D., Batson, J.G., Todd, R.M., Brummett, B.H., Shaw, L.L., \& Aldeguer, C.M.R. (1995). Empathy and the collective good: caring for one of the others in a social dilemma. Journal of Personality and Social Psychology, 68 (4), 619-631. doi: 10.1037//0022-3514.68.4.619

Beck, R., Taylor, C., \& Robbins, M. (2003). Missing home: sociotropy and autonomy and their relationship to psychological distress and homesickness in college freshmen. Anxiety, Stress and Coping, 16(2), 155-162. doi: 10.1037/0022.351.68.4.619 
Belmont-Report. (1978). Ethical Principles and Guidelines for the Protection of Human Subjects of Research, Report of the National Commission for the Protection of Human Subjects of Biomedical and Behavioral Research. www.bioeticayderecho.ub.es (accessed 10 February 2012).

Björkqvist, G.V., Österman, K., \& Kaukiainen, A. (2005). Social intelligence-empathy: Agression? Agression and Violent Behavior, 5(2), 191-200. doi:10.1016/S13591789(98)00029-9

Carlo, G., Allen, J.B., \& Buhman, D.C. (1999). Facilitating and disinhibiting prosocial behaviors: The nonlinear interaction of trait perspective taking and trait personal distress on volunteering. Basic and Applied Social Psychology, 21(3), 189-197. doi: $10.1207 / 15324839951036362$

Carr, S., Colthurst, K., Coyle, M., \& Elliott, D. (2012). Attachment dimensions as predictors of mental health and psychosocial well-being in the transition to university. European Journal of Psychology of Education. doi: 10.1007/s10212-012-0106-9

Chan, K.B., Lai, G., Ko, Y.C., \& Boey, K.W. (2000). Work stress among six professional groups: the Singapore experience. Social Science and Medicine, 50(10), 1415-1432. Doi: 10.1016/S0277-9536(99)00397-4

Chandavarkar, U., Azzam, A., \& Mathews, C.A. (2007). Anxiety symptoms and perceived performance in medical students. Depression and Anxiety, 24(2), 103-111. doi: 10.1002/da.20185

Cross, D.I., \& Hong, J.Y. (2012). An ecological examination of teacher's emotions in the school context. Teaching and Teacher Education, 2(7), 957-967. doi: 10.1016/j.tate.2012.05.001

Dahlin, M., Joneborg, N., \& Runeson, B. (2005). Stress and depression among medical students: a cross-sectional study. Medical Education, 39(6), 594-604. doi: 10.1111/j.1365-2929.2005.02176.x

Derogatis, L.R. (1993). BSI Brief Symptom Inventory. Administration, Scoring, and Procedures Manual (4th Ed.). Minneapolis, MN: National Computer Systems.

Derogatis, L.R., \& Spencer, PM. (1982). The Brief Symptom Inventory (BSI): Administration, and Procedures Manual-I. Baltimore, MD: Clinical Psychometric Research.

Dwyer, A.L., \& Cummings, A.L. (2001). Stress, self-efficacy, social support, and coping strategies in university students. Canadian Journal of Counselling, 35(3), 208-220. 
Dyson, R., \& Renk, K. (2006). Freshmen adaptation to university life: depressive symptoms, stress and coping. Journal of Clinical Psychology, 62(10), 1231-1244. doi: 10.1002/jclp.20295

Eisenberg, N., Fabes, R,A., Murphy, B., Karbon, M., Maszk, P., Smith, M., O’Boyle, C., \& Suh, K. (1994). The relations of emotionality and regulation to dispositional and situational empathy-related responding. Journal of Personality and Social Psychology, 66(4), 776-797. doi: 10.1037/0022-3514.66.4.776

Eisenberg, N., Fabes, R.A., Guthrie, I.K., \& Reiser, M. (2000). Dispositional emotionality and regulation: their role in predicting quality of social functioning. Journal Personality and Social Psychology, 78, 136-157. doi: 10.1037/0022-3514.78.1.136

Fickova, E., \& Korcova, N. (2000). Psychometric relations between self-esteem measures and coping with stress. Studia Psychologica, 42(3), 237-242.

Frydenberg, E., Lewis, R., Kennedy, G., Ardila, R., Frindte, W., \& Hannoun, R. (2003). Coping with Concerns: An Exploratory Comparison of Australian, Colombian, German, and Palestinian Adolescents. Journal of Youth and Adolescence, 32(1), 59-66.

Gardner, S. (2010). Stress among prospective Teachers: a review of the literature. Australian Journal of Teacher Education, 35(8), 18-28.

Garlow, S.J., Rosenberg, J., Moore, J.D., Haas, A.P., Koestner, B., Hendin, H., \& Nemeroff, C.B. (2008). Depression, desperation, and suicidal ideation in college students: results from the American Foundation for Suicide Prevention College Screening Project at Emory University. Journal of Depression and Anxiety, 25 (6), 482-488. doi: $10.1002 /$ da. 20321

Granello, P.F. (1999). College students' wellness as a function of social support and empathetic ability. Journal of College Counseling, 2, 110-120. doi: 10.1002/j.21611882.1999.tb00149.x

Hoffman, M.L. (2000). Empathy and moral development. Cambridge: Cambridge University Press.

Holahan, C., Moos, R.H., Holahan, C.K., Brennan, P.L., \& Schutte, K.K. (2005). Stress Generation, Avoidance Coping, and Depressive Symptoms: A 10-Year Model. Journal of Consulting and Clinical Psychology, 73(4), 658-666. doi: 10.1037/002006x.73.4.658

Joshanloo, M., \& Chaedi, G., (2009). Value priorities s predictors of hedonic and eudaimonic aspects of well-being. Personality and Individual Differences. 47(4), 294-298. 
Kaunitz, N., Spokane, A.R., Lissitz, R.W., \& Strein, W.O. (1986). Stress in student teachers: A multidimensional scaling analysis of elicited stressful situations. Teaching and Teacher Education, 2 (2), 169-180. doi: 10.1016/0742-051X(86)90015-6

Kirchner, T., Forns, M., Amador, J.A., \& Muñoz, D. (2010). Stability and consistency of coping in adolescence: a longitudinal study. Psicothema, 22, 382-388.

Kirchner, T., Forns, M., Muñoz, D., \& Pereda N. (2008). Psychometric properties and dimensional structure of the Spanish version of the Coping Responses Inventory - Adult Form. Psicothema, 20, 902-909.

Lazarus, R.S., \& Folkman, S. (1986). Estrés y procesos cognitivos. [Stress and cognitive processes]. Madrid: Martínez Roca.

Leung T, Siu O., \& Spenctor PE. (2000). Faculty stressors, job satisfaction, and psychological distress among university teachers in Hong Kong: the role of locus of control. International Journal of Stress Management, 7, 121-138.

Leung, S.S.K., Mak, Y.W., Chui, Y.Y., Chiang, V.C.L., \& Lee, A.C.K. (2009). Occupational stress, mental health status and stress management behaviors among secondary school teachers in Hong Kong. Health Education Journal, 68 (4), 328-343. doi: $10.1177 / 0017896909349255$

López-Pérez, B., Fernández-Pinto, I., \& Abad-García, F.J. (2008). TECA. Test de Empatía Cognitiva y Afectiva. [Test of Cognitive and Affective Empathy]. Madrid: TEA Ediciones.

Mikulic, I.M., \& Crespi, M.C. (2008). Adaptación y validación del Inventario de Respuestas de Afrontamiento de Moos (CRI-A) para adultos. [Adaptation and validation of the Coping Responses Inventory of Moos (CRI-A) for adults]. Anuario de Investigaciones, $15,305-312$.

Miner, M.H. (2007). Burnout in the first year of ministry: personality and belief style as important predictors. Mental Health, Religion and Culture, 10, 17-29.

Moos, R.H. (1993). Inventario de Respuestas de afrontamiento para adultos. [Coping Responses Inventory-Adult-Form]. Madrid: TEA Ediciones.

Morrison, R., \& O’Connor, R.C. (2005). A meta-analysis for exploring the diverse causes and effects of stress in Teacher. Canadian Journal of Education, 28(3), 458-486.

Oceja, L. (2008). Overcoming Empathy-Induced Partiality: Two rules of Thumb. Basic and Applied social Psychology, 30(2), 176-182. doi: 10.1080/01973530802209236 
Oceja, L., López-Pérez, B., Ambrona, T., \& Fernández-Pinto, I. (2009). Measuring the Individual Differences in the Disposition to Feel Empathy and Distress: The Vicarious Experiences Scale. Psicothema, 21, 171-176.

Parker, J.D.A., \& Endler, N.S. (1992). Coping with coping assessment: A critical review. European Journal of Personality, 6(5), 321-344. doi: 10.1002/per.2410060502

Parsons, A., Frydenberg, E., \& Poole, C. (1996). Overachievement and coping strategies in adolescents males. British Journal of Educational Psychology, 66 (1), 109-114. doi: 10.1111/j.2044-8279.1996.tb01180.x

Plancherel, B., Bolognini, M., \& Halfon, O. (1998). Coping strategies in early and midadolescence: Differences according to age and gender in a community sample. European Psychologist, 3(3), 192-201. doi: 10.1027/1016-9040.3.3.192

Ruipérez, M.A., Ibáñez, M.I., Lorente, E., Moro, M., \& Ortet, G. (2001). Psychometric properties of the Spanish version version of the BSI: Contributions to the relationship between personality and pychopathology. European Journal of Psychological Assessment, 17(3), 241-250. doi: 10.1027//1015-5759.17.3.241

Shanafelt, T.D., \& West, C.P. (2007). The influence of personal and environmental factor on professionalism in medical education. BMC Medical Education, 7, 29. doi: $10.1186 / 1472-6920-7-29$

Shanafelt, T.D., West, C., Zhao, X., Novotny, P., Kolars, J., Habermann, T., \& Sloan, J. (2005). Relationship between increased personal well-being and enhanced empathy among internal medicine residents. Journal of General Internal Medicine, 20(7), 599564. doi: 10.1111/j.1525-1494.2005.0108.x

Thomas, M.R., Dyrbye, L.N., Huntington, J.L., Lawson, K.L., Novotny, P.J., Sloan, J.A., \& Shanafelt, T.D. (2007). How distress and well-being relate to medical student empathy? A multicenter study. Journal of General Internal Medicine, 22 (2), 177-183. doi: 10.1007/s11606-006-0039-6

Travers, C.J., \& Cooper, C.L. (1996). Teacher under pressure. Stress in the teaching profession. London: Routledge.

University of Barcelona. (2010). Code of Good Research Practices. Barcelona: University of Barcelona.

Vitaglione, G.D., \& Barnett, M.A. (2003). Assessing a new dimension of empathy: empathic anger as a predictor of helping and punishing desires. Motivation and Emotion, 27(4), 301-325. 
Watson, D.C., \& Sinha, B. (2008). Emotion regulation, coping, and psychological symptoms. International Journal of Stress Management, 15(3), 222-234. doi: 10.1037/10725245.15.3.222

Werch, C., Bian, H., Moore, M., Ames, S., DiClemente, C., \& Weiler, R. (2007). Brief Multiple Behaviour Interventions in a College Student Health Care Clinic. Journal of Adolescent Health, 41(6), 577-585. doi: 10.1016/j.jadohealth.2007.06.003

Wong, J.G.W.S., Bheung, E.P.T., Chan, K.K.C., Ma, K.K.M., \& Tang, S.W. (2006). Webbased survey of depression, anxiety and stress in first-year tertiary education students in Hong Kong. Australian and New Zealand Journal of Psychiatry, 40 (9), 777-782. doi: 10.1111/j.1440-1614.2006.01883.x 